\title{
Angle-Resonant Stimulated Polariton Amplifier
}

\author{
P. G. Savvidis, ${ }^{1}$ J. J. Baumberg, ${ }^{1}$ R. M. Stevenson, ${ }^{2}$ M. S. Skolnick, ${ }^{2}$ D. M. Whittaker, ${ }^{3}$ and J. S. Roberts ${ }^{4}$ \\ ${ }^{1}$ Department of Physics \& Astronomy, University of Southampton, Southampton SO17 1BJ, United Kingdom \\ ${ }^{2}$ Department of Physics, University of Sheffield, Sheffield S3 7RH, United Kingdom \\ ${ }^{3}$ Toshiba Research Europe Ltd., Cambridge CB4 4WE, United Kingdom \\ ${ }^{4}$ Department of Electronic and Electrical Engineering, University of Sheffield, Sheffield S1 3JD, United Kingdom
}

(Received 30 July 1999)

\begin{abstract}
We experimentally demonstrate resonant coupling between photons and excitons in microcavities which can efficiently generate enormous single-pass optical gains approaching 100. This new parametric phenomenon appears as a sharp angular resonance of the incoming pump beam, at which the moving excitonic polaritons undergo very large changes in momentum. Ultrafast stimulated scattering is clearly identified from the exponential dependence on pump intensity. This device utilizes boson amplification induced by stimulated energy relaxation.
\end{abstract}

PACS numbers: 71.36. $+\mathrm{c}, 42.50 .-\mathrm{p}, 42.65 .-\mathrm{k}, 78.47 .+\mathrm{p}$

The interaction of light with solids to produce absorption, luminescence, and stimulated emission depends in detail on the coupling between electrons and photons. New physical behaviors result from manipulating both electronic and photonic wave functions, for instance, enhancing the optical gain in semiconductors. Semiconductor microcavities have attracted recent interest in exploring resonant light-matter interactions because their strong anticrossing between quantum well excitons and cavity photons creates two mixed polariton states [1]. A key property of such planar microcavities is that the polaritons' critical optical wave vectors can be directly accessed by coupling light at an angle to the cavity $[2,3]$. The exciton-photon mixing strongly deforms the dispersion relations of the quasiparticles. Despite this dispersion, recent papers suggest their optical properties are well described using independent two-level systems embedded in a cavity [4]. More controversially, the bosonic nature of the polaritons has recently been claimed to promote stimulated scattering, allow Bose condensation, and potentially form a boser [3,5-7]. Recent cw measurements [8-10] of photoluminescence have attributed nonlinearities in the emission rates and shifts in the emission energy to this stimulated scattering.

In this paper we show strong evidence for the presence of a stimulated scattering process, which is, however, different from those previously discussed. This is achieved by using two different angles to separately pump and initiate the process. The scattering provides a new approach to generating gain in semiconductors, manifest in the amplification of a probe pulse by nearly 2 orders of magnitude in a single transit, when the microcavity is pumped at a resonant angle. Modification of the polariton dispersion is crucial to the scattering, which is forbidden for excitons. Systematic studies as a function of detuning show that two propagating pump polaritons scatter to release a stationary pump polariton providing gain, and a high-k partner polariton to conserve energy and momentum [Fig. 1(a)]. In contrast to previous work, the exciton-polariton remains well defined throughout the gain process, and recovers to its original state after the pulses have passed.

Our motivation for simultaneous time- and angleresolved experiments is to directly track the postulated stimulated scattering process. By injecting and probing polaritons at particular points along the dispersion relation, both energy and momentum scattering is observed on ultrafast time scales. Construction of a femtosecond-stable goniometer allows angular tuning up to $\theta \leq \pm 80^{\circ}$ with sub-ps time shifts. Together with spectral filtering of both pump and probe femtosecond pulses, this provides access to the relevant range of the polariton dispersion relation.

The semiconductor microcavity sample [Fig. 1(b)] is grown by metalorganic vapor-phase epitaxy and consists of two pairs of three $100 \AA \mathrm{In}_{0.06} \mathrm{Ga}_{0.94}$ As quantum wells in $100 \AA$ GaAs barriers, sandwiched between 17 (20) pairs of distributed Bragg reflectors $\mathrm{GaAs} / \mathrm{Al}_{0.18} \mathrm{Ga}_{0.82} \mathrm{As}$ on top (bottom). The optical cavity length is $\sim 3 \lambda_{\mathrm{ex}} / 2$ and varies across the sample allowing access to both positive and negative detunings, $\Delta=\omega_{\text {ex }}-\omega_{\text {cav }}(\theta=0)$, of
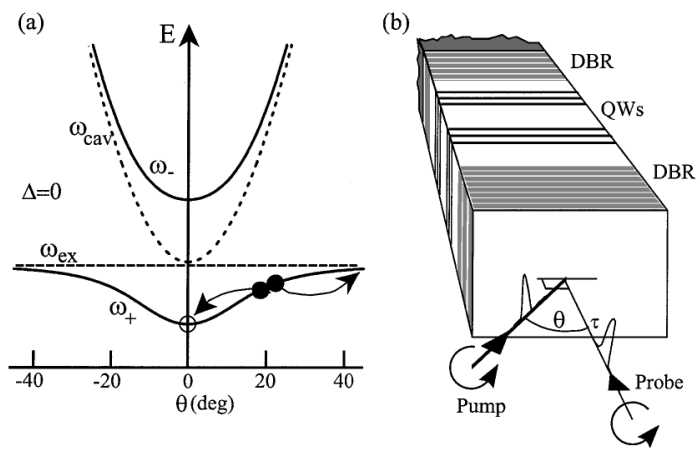

FIG. 1. (a) Polariton dispersion relation vs incident angle $\theta$ at zero detuning. Cavity (exciton) energies $\omega_{\text {cav }}\left(\omega_{\text {ex }}\right)$ shown dashed. Probe polariton ( $\circ$ ) stimulates the scattering of pump polaritons $(\bullet)$. (b) Sample structure and experimental geometry probed at normal incidence and time delay $\tau$, while changing the pump angle $\theta$. 
the cavity resonance $\omega_{\text {cav }}$ from the exciton energy $\omega_{\text {ex }}$. The sample is held in a cold-finger, wide field-of-view cryostat at a temperature of $4 \mathrm{~K}$. The cavity mode can be further tuned by changing the angle of incidence, producing the polariton dispersion, $\omega_{ \pm}=\left\{\omega_{\mathrm{ex}}+\omega_{\mathrm{cav}}(\theta) \mp\right.$ $\left.\sqrt{\left[\omega_{\text {ex }}-\omega_{\text {cav }}(\theta)\right]^{2}+\Omega^{2}}\right\} / 2$, where $\Omega$ is the excitonphoton coupling strength, which is shown in Fig. 1(a) for resonance at normal incidence $(\Delta=0)$. The sample is resonantly excited by pulses derived from a 100 fs modelocked Ti:sapphire laser. Pump and probe pulses are separately spectrally filtered inside zero-dispersion grating compressors using a computer-controlled spatial light modulator [11] or slit, in order to selectively excite the upper or the lower polariton branch. Polarization of the incident beams is set by tunable wave plates calibrated to compensate for Fresnel losses.

The scheme of Fig. 1(a) is investigated by injecting up to $2 \mathrm{~mW}$ pump pulses of $1.5 \mathrm{meV}$ bandwidth at specific angles to the sample growth axis, onto a $100 \mu \mathrm{m}$ spot. To measure the response of the lowest energy polariton states, a broad band 100 fs probe pulse $(<0.3 \mathrm{~mW})$ is focused along the growth axis to a $50 \mu \mathrm{m}$ spot on the sample, and the specular backreflected light collected. In the absence of the pump, the reflected probe spectrum shown in Fig. 2(a) shows the two polariton states split by the normal mode splitting $\hbar \Omega \sim 7 \mathrm{meV}$. This spectrum is radically modified when the pump pulse arrives, provoking narrow band gains around the lower polariton of up to $70(\Delta R / R=7000 \%)$. These enormous gains for the injected pulses are observed when the pump (lower spectra) is detuned above the $\omega_{+}(\theta=0)$ polariton by $2 \mathrm{meV}$ and its incident angle is $16.5^{\circ}$. Thus the amplified probe can be clearly distinguished from scattered pump photons, indicating the involvement of energy loss in this process.

To confirm the gain is real and not spectral conversion of photons within the probe spectrum, a narrow bandwidth probe centered at the lower polariton is used, producing the amplified spectrum shown in Fig. 2(a) (inset). This emission is shifted $0.8 \mathrm{meV}$ above the lower polariton. In the absence of the probe pulse, the pump luminescence collected in the same direction is over 2 orders of magnitude weaker. The enormous amplification of the spectrally narrow probe beam can be recorded on a photodiode as a function of the time delay after the pump pulse arrives [Fig. 2(b)]. The gain builds up over a few ps as the pump pulse arrives (injecting $\sim 5.10^{11}$ photons pulse $\mathrm{sm}^{-1} \mathrm{~cm}^{-2}$ ) and turns off sharply after it passes. This high-gain submicron device stretches the amplified pulse length by $<50 \%$. The temporal response is spectrally resolved in Fig. 2(c), clearly demonstrating that transient narrow band gain exists without destroying the upper polariton resonance, and the recovery is immediate. This is in stark contrast to previous reports $[8,12-14]$ which use power densities over an order of magnitude larger, achieving at best gains of 2, and rapidly washing out all traces of exciton-polaritonic coupling.
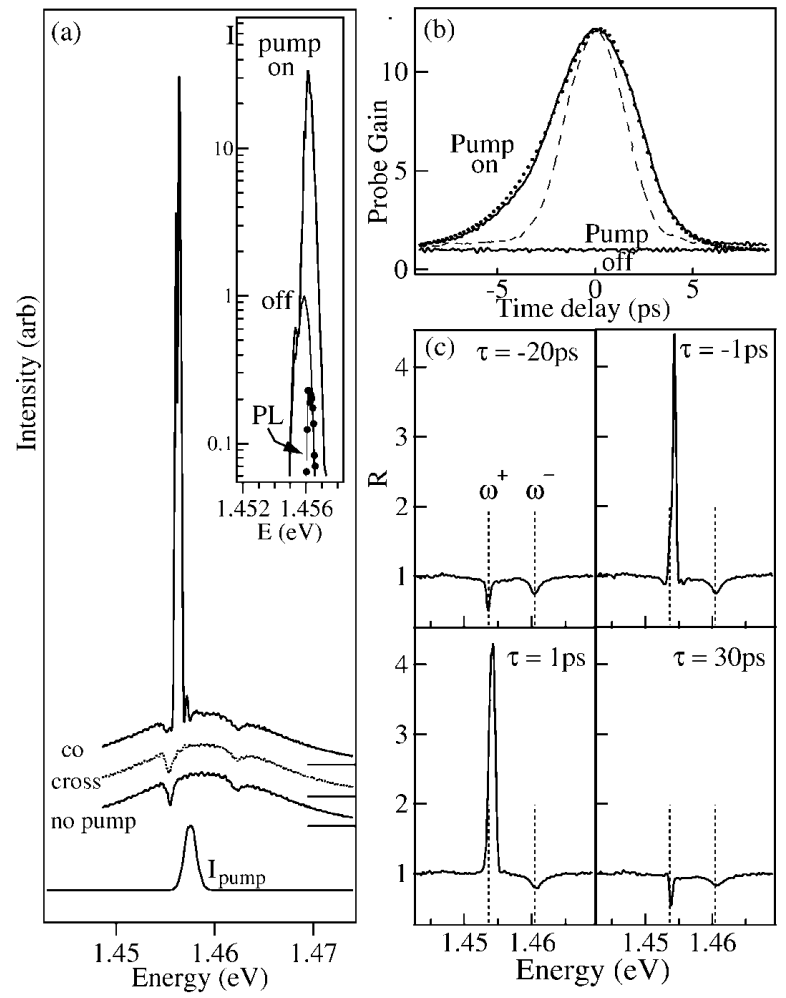

FIG. 2. (a) Reflected probe spectra at $\tau=0$ ps for pump off, co-, and cross-circularly polarized to the probe. Spectral oscillations are caused by interference between reflections from front and back of the sample. Pump spectrum on lower trace. Inset:

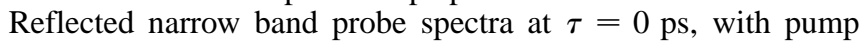
pulse on/off, together with pump PL without probe pulse $(\bullet)$. (b) Reflected probe power vs time delay, with and without pump. Temporal resolution shown dashed and theoretical fit dotted. (c) Probe reflection spectra at several time delays. Pump powers are (a),(b) $22 \mathrm{~W} / \mathrm{cm}^{2}$ and (c) $4.5 \mathrm{~W} / \mathrm{cm}^{2}$ and $\Delta=0$.

Previous explanations for nonlinearities within semiconductor microcavities have implicated four wave mixing modeled with static excitons [15]. In this picture the fermion constituents of the exciton and their Coulomb couplings are responsible for the observed interactions. A number of recent discussions $[4,13]$ also stress the successful treatment of microcavity phenomena using cavity photons coupling to simple two-level systems (i.e., excitons). The data presented here, however, require a full polaritonic treatment based on the reshaped dispersion relations and the new behavior of the polariton quasiparticles.

Strong evidence for polariton scattering is provided by changing the incident angle of the pump pulse. The pump energy is simultaneously tuned for maximum gain, which occurs when resonantly exciting the lower polariton branch. We map out a sharp resonant enhancement around a critical angle which depends on the detuning of the exciton and photon modes. For zero detuning $(\Delta=0)$, gain is observed only for $\theta=16.5 \pm 2^{\circ}$, and is totally absent in the conventional near-normal incidence geometry at these moderate pump intensities (Fig. 3). The critical angle for gain is an unambiguous signature that 


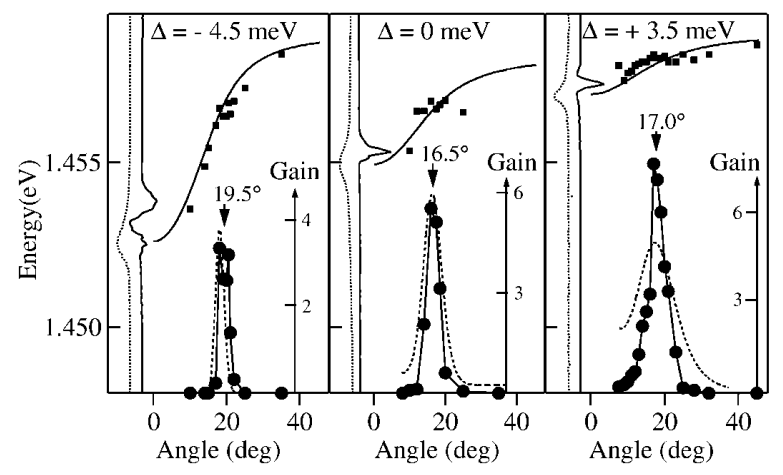

FIG. 3. Parametric gain $(\bullet)$ vs angle for detunings

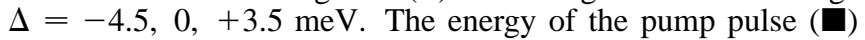
is tuned to achieve maximum gain. Fitted dispersion relations (lines) match the reflected probe spectra shown solid (dashed) on the left side for pump on (off). The gain peaks are at $19.5 \pm 1.5^{\circ}, 16.5 \pm 2^{\circ}$, and $17 \pm 2.5^{\circ}$, respectively, compared to the predicted curves shown dashed, which are at $18.0^{\circ}, 16.3^{\circ}$, and $17.2^{\circ}$. Pump (probe) powers are $20 \mathrm{~W} / \mathrm{cm}^{2}\left(0.4 \mathrm{~W} / \mathrm{cm}^{2}\right)$.

specific energy-momentum scattering is needed for the gain process. This would not occur in four wave mixing, for which interfering polaritons at any two angles can cause nonlinear diffraction.

This strongly suggests the dominance of the previously overlooked scattering process shown in Fig. 1(a). Two $\mathbf{k}_{\mathbf{p}}$ polaritons injected by the pump are stimulated by a probe polariton at $\mathbf{k}=0$, to scatter into polaritons at $2 \mathbf{k}_{\mathbf{p}}$ and $\mathbf{0}$. Energy and momentum conservation restrict the range of $\mathbf{k}_{\mathbf{p}}$ to those satisfying $E\left(\mathbf{2} \mathbf{k}_{\mathbf{p}}\right)-E\left(\mathbf{k}_{\mathbf{p}}\right)=$ $E\left(\mathbf{k}_{\mathbf{p}}\right)-E(\mathbf{0})$. The predicted angular resonances satisfying this condition (Fig. 3, dashed line) are based on the measured polariton linewidths and dispersions and are in excellent agreement with the data. In particular, the model successfully accounts for the increase in resonant $\theta$ at both positive and negative detunings. It also reproduces the narrower width of the angular resonance as the detuning becomes more negative, due to the increased curvature of the lower polariton dispersion. The energy dispersion of acoustic phonons [8] is unable to account for these data. In a simplistic picture, one of two moving polaritons escapes by the probe-enhanced conversion, while the other carries off the energy and momentum. This high-k polariton is detected in emission at $35^{\circ}$ and will be reported in detail elsewhere. The scattering condition is impossible to satisfy for the quadratic dispersion provided by either the exciton or cavity modes alone and proves the involvement of polariton quasiparticles.

The scattering process is stimulated by probe polaritons, as expected for the hypothesis of bosonic scattering (which previously considered a different scheme) $[3,5,8,9]$. The strong polariton coupling masks the normally dominant fermionic nature of the carriers, thus favoring scattering which is enhanced by occupation of the final state. Injecting probe photons stimulates scattering into this final state $N_{\text {final }}$, since for bosons $\frac{d N_{\text {final }}}{d t} \propto\left(1+N_{\text {final }}\right) N_{\text {pump }}$, for a bath of $N_{\text {pump }}$ pump polaritons. This dependence can also be obtained [16] by integrating Maxwell's equations coupling the boson field amplitudes through $\frac{\partial E_{\text {probe }}}{\partial t} \propto E_{\text {probe }}\left|E_{\text {pump }}\right|^{2}$. Numerical simulation of the evolving polariton densities using these rate equations and incident pulse profiles (without adjustable parameters) reproduces the observed asymmetric time dependence of the gain [Fig. 2(b), dotted line]. The polariton density injected into each mode is given by the number of photons/pulse divided by the number of $\mathbf{k}$ states excited. For a 2 fJ probe pulse with photon density $P \sim 4 \times 10^{8} \mathrm{~cm}^{-2}$ focused down to an acceptance angle $\phi \sim 10^{-2} \mathrm{rad}$ at the sample, $N_{\text {final }}=P /\left[\pi\left(\phi / \lambda_{\text {ex }}\right)^{2}\right] \sim 10^{4}$ polaritons $/$ state $(\gg 1)$. As expected, the emerging probe power is found to be almost linear with the injected probe power: $I_{\text {probe }}^{\text {out }} \propto\left(I_{\text {probe }}^{\text {in }}\right)^{0.8}$, while saturating at higher probe powers [Fig. 4(a)]. The power gain (inset) drops as the probe power increases, producing maximum transfer efficiencies from pump to probe of order $5 \%$.

Stimulated scattering is confirmed experimentally by the observed exponential dependence of gain on the pump power [Fig. 4(b)], although at intensities above $20 \mathrm{~W} / \mathrm{cm}^{2}$
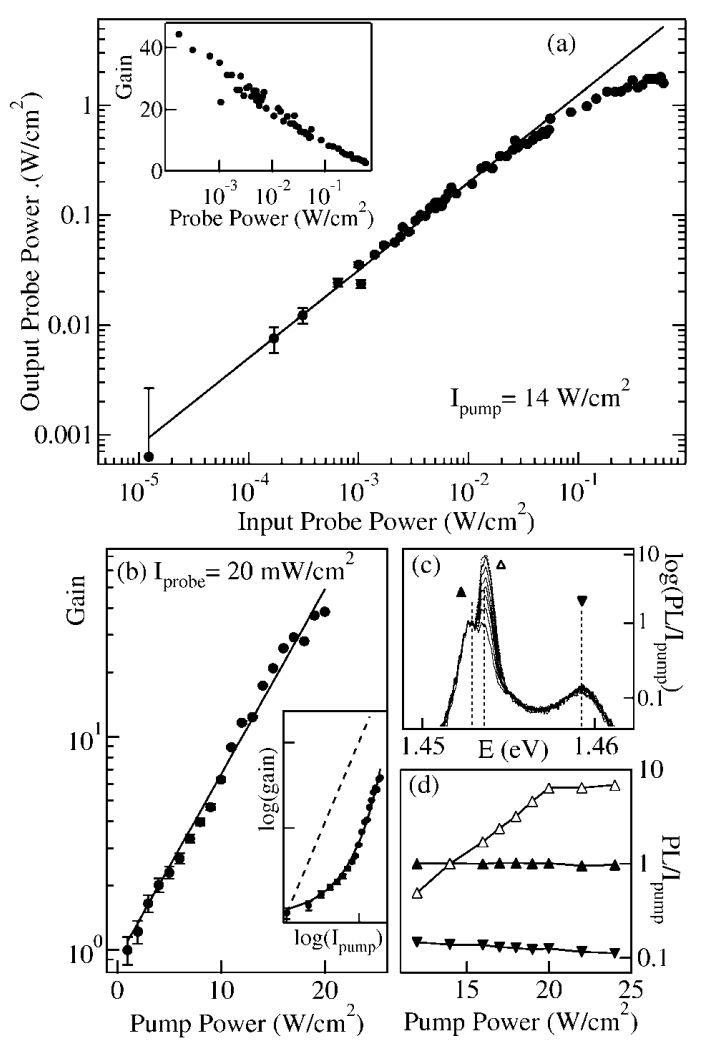

FIG. 4. (a) Reflected vs incident probe power for a pump power of $14 \mathrm{~W} / \mathrm{cm} 2$. Fit: $I_{\text {probe }}^{\text {out }} \propto\left(I_{\text {probe }}^{\text {in }}\right)^{0.8}$. Inset: Probe gain vs probe power. (b) Semilog plot of probe gain vs incident pump power. Inset: $\log$ plot—-dashed line $\propto I_{\text {pump }}^{2}$ while solid curve $\propto \exp \left(g I_{\text {pump }}\right)$. (c),(d) Normalized spontaneous PL emission from the pump alone vs pump power, for $\Delta=+1.5 \mathrm{meV}$. Emission collected at normal incidence with detection cone of $\pm 1^{\circ}$ showing relative emission at the upper $(\boldsymbol{\nabla})$, lower $(\boldsymbol{\Delta})$ polariton and extra peak on higher energy side of lower polariton $(\triangle)$. 
some saturation occurs. This exponential behavior is expected because the boson scattering rate depends on the number of pump polaritons (integrated over the pulse duration), so $N_{\text {final }} \propto\left(1+N_{0}\right) \exp \left(g I_{\text {pump }}\right)$. Conventional four wave mixing usually produces a quadratic pump power dependence [shown dashed in Fig. 4(b), inset] which is unable to fit our data. Because of this exponential pumping, the slope efficiency approaches $1000 \%$ at low probe powers. For comparison, Figs. 4(c) and 4(d) show the spontaneous emission from the pump excitation alone, collected in the probe direction and normalized to the pump power. The spectra clearly reveal a new photoluminescence (PL) peak appearing $0.8 \mathrm{meV}$ above the lower polariton for intensities above $10 \mathrm{~W} / \mathrm{cm}^{2}$. The PL exhibits stimulated gain and resembles nonlinear luminescence seen in recent cw photoluminescence measurements $[8,9]$. Using pulsed illumination the new state is observed more clearly and at significantly lower powers. The use of ultrafast time- and angle-resolved techniques is crucial for identifying the new scattering process actually responsible.

The postulated scattering process seen in Fig. 2(a) is highly spin dependent and relies on cocircular polarizations of the pump and probe polaritons. It is completely suppressed for opposite circular polarizations, and at these low powers exhibits negligible nonlinearities for the opposite spin polaritons, confirming the absence of exciton bleaching. Correspondingly, linearly polarized pump and probe beams produce almost identical gain irrespective of their orientation showing that the spin selection rules for excitons in the quantum wells dominate. In addition, the new PL peak, which appears in Fig. 4(c) for the pump pulse alone, is completely spin polarized in contrast to the partial depolarization found at the upper and lower polariton energies. Additional evidence that this peak corresponds to the appearance of new bound quasiparticles comes from the blueshift between lower polariton and gain peak which is independent of power.

The observed gain differs from conventional $\chi^{(3)}$ processes, most obviously in the exponential dependence on pump power. It can be seen as optical parametric amplification, based on a coherent $\chi^{(3)}$ instead of $\chi^{(2)}$, which is resonantly enhanced by engineering the microcavity dispersion. Coherent $\chi^{(3)}$ processes in semiconductors are normally weak and masked by incoherent contributions such as bleaching of absorption. Instead of conventional phase matching from nonresonant photon mixing, here energy-momentum conservation demands the high-k polariton to balance the process.

In summary, we report the observation of giant singlepass optical gain by pumping semiconductor microcavities at specific angles. A key innovation is the use of excitation and probe pulses at different angles to identify the scattering. The predicted angles from energy-momentum conservation of polariton fission match well to the experiment. The ability to deform polariton dispersions provides a new path to resonant nonlinear phenomena in these systems.

J. J. B. enthusiastically acknowledges discussions with Benoit Deveaud, Stefan Koch, and the support of the University of Southampton, Coherent U.K. Ltd., and Hitachi Europe Ltd. This work was partly supported by EPSRC GR/M43890, GR/L32187, and HEFCE JR98SOBA.

[1] C. Weisbuch, M. Nishioka, A. Ishikawa, and Y. Arakawa, Phys. Rev. Lett. 69, 3314 (1992).

[2] M.S. Skolnick, T. A. Fisher, and D. M. Whittaker, Semicond. Sci. Technol. 13, 645 (1998).

[3] R. Huang, F. Tassone, and Y. Yamamoto, Microelectron. Eng. 47, 325 (1999).

[4] C. Ell et al., Phys. Rev. Lett. 80, 4795 (1998).

[5] A. Imamoḡlu et al., Phys. Rev. A 53, 4250 (1996).

[6] M. Kira et al., Phys. Rev. Lett. 79, 5170 (1997).

[7] F. Tassone and Y. Yamamoto, Phys. Rev. B 59, 10830 (1999); A. I. Bobrysheva et al., Phys. Status Solidi (b) 212, 105 (1999).

[8] P. Senellart and J. Bloch, Phys. Rev. Lett. 82, 1233 (1999).

[9] Le Si Dang et al., Phys. Rev. Lett. 81, 3920 (1998).

[10] S. Pau et al., Phys. Rev. A 54, R1789 (1996).

[11] J. J. Baumberg, A. Armitage, M.S. Skolnick, and J.S. Roberts, Phys. Rev. Lett. 81, 661 (1998).

[12] F. Quochi et al., Phys. Rev. Lett. 80, 4733 (1998).

[13] F. Quochi et al., Phys. Rev. B 59, R15 594 (1999).

[14] J.-K. Rhee, D. S. Citrin, T. B. Norris, Y. Arakawa, and M. Nishioka, Solid State Commun. 97, 941 (1996).

[15] M. Kuwata-Gonokami et al., Phys. Rev. Lett. 79, 1341 (1997); M. Shirane et al., Phys. Rev. B 58, 7978 (1998).

[16] Y.R. Shen, The Principles of Nonlinear Optics (Wiley, New York, 1984). 